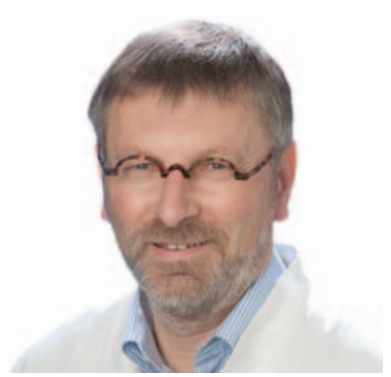

\title{
Die alleinige Bestimmung der Sauerstoffsättigung kann nicht die Patienten identifizieren, die von einer Langzeitsauerstoff-Therapie profitieren
}

\author{
Dr. Peter Haidl \\ Fachkrankenhaus Kloster Grafschaft GmbH, Abteilung Pneumologie II
}

Abstractübersetzung aus Jung DW, Hwang SH, Lee YJ, Jeong D-U, Park KS: Oxygen desaturation index estimation through unconstrained cardiac sympathetic activity assessment using three ballistocardiographic systems. Respiration 2016;92:90-97.

Einschätzung des Sauerstoff-Entsättigungsindex durch kontaktlose Messung der kardialen sympathischen Aktivität mittels dreier Ballistokardiografie-Systeme

\section{Schlüsselwörter}

Nächtliche Hypoxämie · Sauerstoff-Entsättigungsindex · Kardiale sympathische Aktivität · Herzfrequenzvariabilität Ballistokardiografie

\section{Zusammenfassung}

Hintergrund: Nächtliche Hypoxämie, gekennzeichnet durch anomal niedrige Sauerstoffsättigungswerte im arteriellen Blut während des Schlafs, ist ein wichtiges Merkmal verschiedener Gesundheitsstörungen. Der Sauerstoff-Entsättigungsindex, ein gängiges Maß für den Schweregrad der nächtlichen Hypoxämie, wird mittels nächtlicher Pulsoxymetrie ermittelt. Hierzu muss der Patient über Nacht einen Pulsoxymeter-Sensor tragen.

Ziele: Ziel der Studie war es, eine mögliche Methode zur kontaktlosen Einschätzung des Entsättigungsindex zu erarbeiten.

Methoden: Wir stellten die Hypothese auf, dass die Schwere der nächtlichen Hypoxämie positiv mit der sympathischen Aktivierung des Herzens während des Schlafs assoziiert ist. Mittels dreier unterschiedlicher Ballistokardiografie-Systeme erfolgte eine kontaktlose Überwachung der Herzfrequenzvariabilität, um die kardiale sympathische Aktivität zu beurteilen. Wir erfassten gepaarte polysomnografische und ballistokardiografische Aufzeichnungen von 20 Personen mit nicht-nächtlicher Hypoxämie (Sauerstoff-Entsättigungsindex $<5$ Ereignisse/h) und 76 Personen mit nächtlicher Hypoxämie. Von diesen 96 gepaarten Aufzeichnung wurden 48 als Trainingsdaten verwendet und die anderen 48 als Testdaten.

Ergebnisse: Die Regressionsanalyse des Niedrigfrequenzbereichs der Herzfrequenzvariabilität ergab eine mittlere quadratische Abweichung von 3,33 Ereignissen/h zwischen den ermittelten Schätzwerten und den Referenzwerten des Sauerstoff-Entsättigungsindex. Die nächtliche Hypoxämie-Diagnostik nach unserem Verfahren zeigte eine durchschnittliche Güte von 96,5\% bei Cutoff-Werten für den Sauerstoff-Entsättigungsindex von $\geq 5$, 15 und 30 Ereignissen/h.

Schlussfolgerungen: Unsere Methode zeigt Potenzial als ergänzende Maßnahme für den Fall des zufälligen Abrutschens eines Pulsoximeter-Sensors bei der nächtlichen Pulsoxymetrie. Die unabhängige Anwendung unseres Verfahrens könnte die Langzeitüberwachung des Sauerstoff-Entsättigungsindex im häuslichen Umfeld erleichtern.

(c) 2017 S. Karger GmbH, Freiburg

\section{KARGER}

Fax +497614520714 information@karger.com www.karger.com

\section{(c) 2017 S. Karger GmbH, Freiburg}




\section{Transfer in die Praxis}

\section{Hintergrund}

Die Indikationsstellung zur Sauerstoff-Langzeittherapie (LTOT) bei COPD basiert auf2 Studien, die vor mehr als 30 Jahren durchgeführt wurden. Ausgangspunkt der Verordnung sind pO2-Werte am Tage im stabilen Krankheitsintervall. Einbezogen wird weiter der pO2 unter körperlicher Belastung. Die Bedeutung der nächtlichen Sauerstoffsättigung sowie nächtlicher Desaturationen ist unklar.

\section{Studienergebnisse}

Jung et al. untersuchten ein kontaktloses Analyseverfahren, das Ballistokardiogramm (BKG). Ohne direkten Kontakt zum Patienten können auf einem speziellen Lagerungstisch bzw. einem einzigen Sensor im Patientenbett durch die mechanische Aktivität des Herzens ausgelöste Körperbewegungen aufgezeichnet werden. Dadurch wird die kardiale sympathische Aktivität gemessen. Diese Ergebnisse wurden mit dem nächtlichen Desaturationsindex verglichen, der mittels Pulsoxymetrie erhoben wurde. Es ergab sich eine signifikante Korrelation.

Daher folgerten die südkoreanischen Autoren, dass das BKG als additive Messmethode zur Bestimmung nächtlicher Desaturationen infrage kommt. Vorteil ist die geringe Störanfälligkeit der Methode im Vergleich zur Pulsoxymetrie. Fehlende Messergebnisse z.B. durch Verrutschen des Fingerclips werden vermieden.

\section{Fazit für die Praxis}

\section{Kontextualisierung}

Der Vergleich des BKG mit der Pulsoxymetrie bezüglich der Detektion von nächtlichen Desaturationen ist sicher ein interessanter Aspekt. Im Vordergrund steht letztlich die Frage, welche Gruppe der COPD-Patienten am meisten von einer LTOT profitiert. Hier wurden seit Langem die Ergebnisse der «Long-term Oxygen Treatment Trial Research Group» erwartet, die nun im Oktober 2016 publiziert wurden [1]. Aufgrund spezifischer Gegebenheiten in den USA (ein Teil der Studienzentren hatte kein BGA-Gerät zur Verfügung!) wurde alleine die im stabilen Intervall einmalig gemessene Sauerstoffsättigung zwischen 89 und 93\% als Einschlusskriterium gefordert, d.h. die Werte lagen noch im flachen Teil der Sauerstoffbindungskurve. Des Weiteren wurden Patienten mit einer Sättigung von 94\% und höher aufgenommen, wenn unter Belastung die Sättigung mindestens $10 \mathrm{~s}$ unter $90 \%$ abfiel. Eingeschlossen wurden 738 Patienten mit einem Post-Bronchodilator $\mathrm{FEV}_{1}$ unter 65\% Soll. Die Teilnehmer wurden auf eine Sauerstoffgruppe (O2 über 24 h, Fluss $21 \mathrm{O} 2 / \mathrm{min}$ ) und eine Kontrollgruppe verteilt. Pri- märe Endpunkte waren die Zeit bis zum Tod und die Zeit bis zur ersten Exazerbation. Zusammenfassend ergaben sich durch die LTOT für die genannte Patientengruppe keine signifikanten Vorteile. Die Diskussion lässt mögliche Ursachen dieser Ergebnisse offen und verweist darauf, dass vermutlich erst ab einer Sättigung unter 88\% Effekte auf die pulmonale Vasokonstriktion, die Mediatorfreisetzung und den Atemantrieb klinisch relevant werden.

Was lernen wir aus der Studie der «Long-term Oxygen Treatment Trial Research Group»?

Es konnte nur das bekannte Ergebnis von Gorecka et al. [2] bestätigt werden, dass COPD-Patienten mit einer moderaten Hypoxämie nicht von einer LTOT profitieren. Im Studienansatz ist die Hypothese, dass die Mortalitätssenkung der LTOT auf einer Entlastung der Atemmuskulatur beruhen könnte, in keiner Weise gewürdigt worden. Bereits in der NOTT-Studie [3] war darauf hingewiesen worden, dass Patienten mit einer moderaten Hyperkapnie eine stärkere Mortalitätssenkung erreichen.

Zumindest ist es eine pathophysiologische Grundlage, dass ein pO2 oder die Sättigung stets in Zusammenhang mit der dafür erforderlichen Ventilation zu interpretieren ist. Eine Studie ohne Angabe des pCO2 beschreibt daher das Patientenkollektiv zu ungenau und führt wie in der aktuellen Studie dazu, dass der Subgruppe mit Zeichen einer Ermüdung der Atemmuskulatur eine lebensverlängernde Therapie möglicherweise vorenthalten wird.

\section{Disclosure Statement}

Hiermit erkläre ich, dass keine Interessenskonflikte in Bezug auf den vorliegenden Kommentar bestehen.

\section{Literatur}

1 Long-term Oxygen Treatment Trial Research Group: A randomized trial of long-term oxygen for COPD with moderate desaturation. N Engl J Med 2016;375:1617-1627.

2 Górecka D, Gorzelak K, Sliwiński P, Tobiasz M, Zieliński J: Effect of long-term oxygen therapy on survival in patients with chronic obstructive pulmonary disease with moderate hypoxaemia. Thorax 1997;52:674-679.

3 Nocturnal Oxygen Therapy Trial Group: Continuous or nocturnal oxygen therapy in hypoxemic chronic obstructive lung disease: a clinical trial. Ann Intern Med 1980;93:391-398.

Kontaktadresse: Dr. Peter Haidl, Fachkrankenhaus Kloster Grafschaft GmbH, Abteilung Pneumologie II, Annostraße 1, 57392 Schmallenberg, Deutschland, p.haidl@fkkg.de 